\title{
Perfil de atividade física de usuários da estratégia saúde da família
}

\author{
Physical activity profile of family health strategy users \\ Perfil de la actividad física de usuarios de la estrategia de salud de la familia
}

\author{
Claudia Santos Martiniano'; Sabrina Talita Teotônio Bezerra ${ }^{\text {II; }}$ Karla Karolline Barreto Cardins ${ }^{\text {III; }}$; \\ Larissa Nayane Braz do Nascimento ${ }^{I V}$; Francisco de Sales Clementino ${ }^{V}$; Cyrus Dalva da Silveira Barros ${ }^{\mathrm{VI}}$
}

\begin{abstract}
RESUMO: O objetivo da pesquisa é avaliar o perfil de atividade física de usuários da estratégia saúde da família. Estudo descritivo, realizado entre outubro de 2010 e fevereiro de 2011. Teve como instrumento uma entrevista aplicada com 386 usuários, idade superior a 20 anos, de uma unidade básica de saúde da família, em Campina Grande, Paraíba. Observou-se que $275(71,24 \%)$ disseram que não praticavam atividade física e $111(28,76 \%)$ realizavam. A adesão à atividade física foi maior entre os sujeitos com ensino superior. Entre os que se exercitam, $91(101 \%)$ realizavam a caminhada; $62(55,88 \%)$ faziam mais de três vezes por semana; $80(72,03 \%)$ gastavam um tempo entre 30 e 60 minutos. Conclui-se que a prática da atividade física não é habitual entre eles, devendo ser estimulada pela equipe de saúde.

Palavras-Chave: Atividade física; promoção da saúde; estratégia saúde da família; prevenção de doenças.
\end{abstract}

ABSTRACT: This exploratory, descriptive case study evaluated the physical activity profile of family health strategy users between October 2010 and February 2011. At interview of 386 users over 20 years old, at a family health primary care facility in Campina Grande, Paraíba, 275 (71.24\%) said they did no exercise, while 111 (28.76\%) exercised. Adherence to physical activity was greater among users with higher education. Of participants who did physical exercise, 91 (101\%) walked, 62 $(55.88 \%)$ exercised three times a week, and $80(72.03 \%)$ exercised for 30 to 60 minutes. It was concluded that physical activity is not habitual among users, and should be encouraged by the health team.

Keywords: Physical activity; health promotion; family health strategy; disease prevention

RESUMEN: El objetivo de la investigación es evaluar el tipo de actividad física de los usuarios de la estrategia salud de la familia. Es un estudio descriptivo, realizado entre octubre de 2010 y febrero de 2011 cuyo instrumento ha sido una entrevista aplicada a 386 usuarios, mayores de 20 años, de una unidad básica de salud de la familia en Campina Grande, Paraíba. Se ha observado que $275(71,24 \%)$ declararon que no practicaban actividad física y $111(28,76 \%)$ que sí. La adhesión a la actividad física es más significativa entre individuos con educación superior. Entre los que hacían ejercicio físico, 91 (101\%) caminaban, $62(55,88 \%)$ lo hacían tres veces por semana, $80(72,03 \%)$, gastaban de 30 a 60 minutos en la actividad. Se concluye que la práctica de actividad física es poco común entre los usuarios y debe ser estimulada por el equipo de salud. Palabras Clave: Actividad física; promoción de la salud; estrategia de salud familiar; prevención de enfermedades.

\section{INTRODUÇÃO}

A prática de atividade física esteve presente desde os primórdios da humanidade e, de acordo com a história de sua origem, percebe-se sua presença desde o período pré-histórico, no qual o homem, para sua sobrevivência, realizava caças, corridas e lutas, ou seja, era ativo fisicamente. Com a evolução do homem, a atividade física começou a ser empregada na modalidade de ginástica, fato bem evidente na Antiga Grécia, com os relatos da história, treinamentos para guerras e gladiadores, jogos olímpicos, entre outros. No início da Idade Contemporânea, surge na Europa a atividade física nas modalidades de dança, jogos e ginástica ${ }^{1}$.

A partir dessas práticas, foram desenvolvidos vários métodos de exercícios físicos em diversos locais do mundo, como no Brasil, que tiveram seu início em orientações médicas, valorizando um indivíduo saudável, proporcionando-lhe uma boa aparência física e melhorando sua postura ${ }^{1}$.

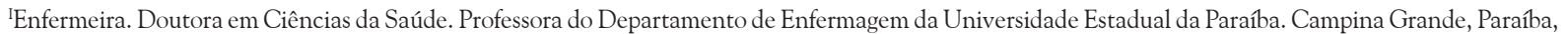
Brasil.E-mail: cmartiniano@ibest.com.br.

IIEnfermeira. Especialista em Enfermagem do Trabalho. Campina Grande, Paraíba, Brasil. E-mail: sabrina_talita@msn.com.

"IIEnfermeira. Mestranda do Programa de Pós-Graduação em Saúde Pública da Universidade Estadual da Paraíba. Campina Grande, Paraíba, Brasil. E-mail: karla karolline@hotmail.com.

IVEnfermeira. Especialista em Saúde da Família. Campina Grande, Paraíba, Brasil.E-mail: larissanayaneb@gmail.com.

vEnfermeiro. Doutor em Enfermagem. Professor do Departamento de Enfermagem da Universidade Federal de Campina Grande. Campina Grande, Paraíba, Brasil. E-mail: fclementino67@yahoo.com.br.

VIMédica da Estratégia Saúde da Família. Campina Grande, Paraíba, Brasil. E-mail: cyrusdalva@hotmail.com. 
Apesar de estudos comprovarem que a prática de atividade física é essencial para uma vida saudável, melhorando a qualidade de vida e diminuindo os índices de doenças crônicas ${ }^{2-5}$, sabe-se que mais de $60 \%$ da população adulta mundial não realizam exercícios físicos de maneira regular ${ }^{6}$.

Isso é resultado de um novo estilo de vida, no qual as pessoas, pela necessidade de se adequarem ao modelo de vida do mundo contemporâneo, estão em busca constante de um aprimoramento profissional, tentando atingir melhores condições de trabalho e remuneração. Como consequência, o tempo que elas possuem para realizar atividades extras não vem sendo ocupado por exercícios físicos, pois como os trabalhos são desgastantes, são preferíveis as atividades que proporcionam relaxamento, como: assistir televisão, sair com a família, navegar na internet ou, simplesmente descansar? ${ }^{7}$ Com isso, pode-se inferir que a falta de tempo é o principal fator de fracasso na adoção de um modelo regular de exercícios ${ }^{8}$. Por esse motivo, a inatividade física tem sido considerada como fator de risco ao lado do fumo, diabetes, hipertensão arterial e dislipidemia?.

Por definição, atividade física é todo e qualquer movimento produzido em consequência da contração muscular que resulte em gasto de energia e alterações do organismo, por meio de exercícios que envolvam movimentos corporais, com aplicação de uma ou mais aptidões físicas, além de atividades mentais e sociais, de modo que terá como resultados os benefícios à saúde ${ }^{10}$. A atividade física pode ser classificada em aeróbica e anaeróbica; a primeira é de baixa resistência, e a segunda, de alta resistência. Ambas causam a diminuição da pressão arterial; no entanto, recomenda-se a atividade aeróbica, pois o efeito na pressão pós-atividade, tanto agudo quanto crônico, é mais intenso nesse tipo de atividade ${ }^{11}$.

Um estilo de vida sedentário interfere na qualidade de vida do indivíduo, podendo, associado a outros fatores, produzir efeitos deletérios nas suas condições de saúde, como o aumento da incidência de diabetes e de doenças cardíacas, contribuindo, portanto, para a elevação da morbimortalidade por doenças crônicas ${ }^{9}$. Há, ainda, uma relação positiva entre atividade física e sono, sendo a prática do primeiro um fator favorável à melhoria da qualidade deste último ${ }^{12}$.

Portanto, a prática de atividades físicas acarreta benefícios biopsicossociais, envolve o ser humano como um todo, proporciona um bem-estar geral do indivíduo e atua tanto na prevenção de doenças como no tratamento e no controle de certas patologias, principalmente as crônicas ${ }^{4}$.

É de fundamental importância que as estratégias de saúde da família (ESF) organizem ações de estímulo às práticas corporais para a população, e que essas ações sejam inseridas onde elas ainda não existem. Nesse sentido, esta pesquisa visa responder a seguinte questão: Qual o perfil de atividade física dos usuários da unidade básica saúde família (UBSF) das Malvinas I?

Considerando a importância do exercício físico para a saúde, torna-se primordial a realização de pesquisas que visem identificar o padrão de atividade física da comunidade, para que seja possível estabelecer sua provável correlação com os agravos à saúde. Além disso, deve-se observar a implantação da Política Nacional de Promoção da Saúde como relevante estratégia do setor para melhorar a qualidade de vida da população, sendo o objetivo deste estudo avaliar o perfil de atividade física dos usuários da ESF.

\section{REVISÃo DE LITERATURA}

Estudos mostram que pessoas que mantêm seus corpos em forma, utilizando meios para se exercitar e controlar o peso, têm o benefício de prolongar a vida. Em especial, entre 50 e 70 anos, as pesquisas constatam que a mortalidade era três vezes menor nas pessoas que estavam em boas condições físicas e as razões para este fato são: a manutenção da forma física e controle do peso, reduzindo bastante as doenças coronarianas, pois resulta na preservação de pressões abaixo do nível limítrofe da normalidade (140x90 mmHg) e redução dos níveis de colesterol total e das lipoproteínas de baixa densidade, assim como o aumento das lipoproteínas de alta densidade. Essas mudanças, em conjunto, ajudam a diminuir o número de ataques cardíacos e acidentes vasculares cerebrais $^{13}$.

Além das razões já citadas, a prática de exercícios propicia o aumento das reservas corporais que podem ser utilizadas quando o organismo adoece. Isso é importante para a preservação da vida quando pessoas idosas desenvolvem alguma patologia como pneumonia, que pode rapidamente requisitar toda reserva respiratória disponível. Além disso, a capacidade de aumentar o débito cardíaco quando se precisa é frequentemente $50 \%$ maior nos idosos que estão em forma ${ }^{13}$.

Várias instituições e organizações tais como a International Federation of Sports Medicine, a American Heart Association, a Organização Mundial de Saúde (OMS) e o American College of Sports Medicine têm enfatizado a importância da adoção de atividade física regular para a melhoria dos níveis de saúde individual e coletiva, especialmente para a prevenção e reabilitação da doença cardiovascular ${ }^{14}$.

Os benefícios das atividades corporais não se resumem apenas à saúde física. Eles abrangem também a saúde mental, ajudando na regulação das substâncias relacionadas ao sistema nervoso, melhoram o fluxo de sangue para o cérebro, bem como a capacidade de lidar 
com problemas e com o estresse, além de auxiliar a manutenção da abstinência de drogas e na recuperação da autoestima e no tratamento da depressão ${ }^{6}$.

No âmbito social do indivíduo, tais atividades melhoram as relações tanto no ambiente de trabalho quanto no familiar. Entre os diversos benefícios que proporcionam à saúde, a atividade física tem sido considerada uma forma de favorecer as capacidades funcionais de todas as pessoas que a praticam ${ }^{6}$.

Segundo a OMS, a participação em atividades físicas leves e moderadas pode também retardar os declínios funcionais. Assim, uma vida ativa melhora a saúde mental e contribui na gerência de desordens como a depressão e a demência ${ }^{15}$.

A predominância do sedentarismo entre a prevalência dos fatores de risco associados à hipertensão arterial é a demonstração eloquente da importância dos exercícios físicos, sobretudo nas elevações menos acentuadas da pressão arterial sem lesões de órgãos-alvo e comorbidades, tais como o Diabetes Mellitus ou nefropatias. Nessas condições, a estratégia de modificação do estilo de vida caracteriza-se como procedimento preferencial para a abordagem da hipertensão arterial ${ }^{6}$.

Nesse sentido, evidencia-se a necessidade de recomendação da prática de atividade física por parte dos profissionais de saúde, com o objetivo de prevenir e controlar doenças e evitar o risco de morte por problemas cardíacos e promover a melhora na qualidade de vida da população ${ }^{4}$.

Os exercícios físicos interferem na redução da pressão arterial pelo envolvimento de fatores hemodinâmicos, humorais e neurais ${ }^{6}$. $\mathrm{O}$ efeito antihipertensivo do exercício físico resulta de adaptações neuro-humorais, vasculares e estruturais, resultando em diminuição da resistência vascular periférica, como menor produção de catecolaminas e melhora da sensibilidade à insulina, atenuação da resposta $\alpha$-adrenérgica para norepinefrina, modificação na produção dos vasoconstrictores e vasodilatadores locais e o remodelamento e angiogênese vascular ${ }^{16}$.

A pesquisa sobre sedentarismo deve fazer parte da avaliação da população hipertensa e seu combate é uma das medidas indicadas para reduzir o risco cardiovascular. Estima-se que indivíduos sedentários tenham um risco aumentado de desenvolver hipertensão entre $20 \%$ e $50 \%{ }^{11}$.

O benefício da atividade física na população normotensa é o de prevenção primária da hipertensão; também se observa uma melhora no perfil lipídico e uma sensação de bem-estar físico nas pessoas que têm uma atividade física regular. Esta é uma prática tão importante de promoção da saúde que a sua prática inversa - o sedentarismo - pode ser considerada um problema de saúde pública.

Nesse sentido, atividades como prescrição de exercícios, práticas lúdicas, esportivas e de lazer devem estar presentes na rede básica de saúde, sendo voltadas tanto para a comunidade como um todo quanto para grupos vulneráveis ${ }^{12}$. Assim, incorporar práticas saudáveis que possam trazer benefícios à população é um dos desafios impostos a toda a estrutura da atenção básica.

\section{Metodologia}

Foi realizado estudo descritivo e quantitativo, que teve como campo empírico a UBSF das Malvinas I, no município de Campina Grande-PB, que conta com duas equipes de saúde da família.

A população foi composta pelos usuários cadastrados da unidade que têm idade acima de 20 anos, pois abaixo dessa faixa etária encontram-se, na maioria das vezes, em atividade física escolar. Os critérios de inclusão foram: serem usuários da área de abrangência da UBSF Malvinas I e maiores de 20 anos. Não participaram da pesquisa os usuários que se enquadraram em, pelo menos, um dos seguintes critérios de exclusão: serem portador de deficiência física que possuam limitações para a realização da prática corporal; estarem acamados ou com impossibilitado de caminhar; e serem menores de 20 anos.

Em levantamento prévio, observou-se que existia, na equipe 1 , um total de 1210 usuários. Na equipe 2, havia um total de 938 usuários nessas condições. Desse modo, foi tomada uma amostra estatística estabelecendo um índice de 95\% de confiança e margem de $5 \%$ de erro em cada estrato, o que significa que seriam investigados 292 usuários na equipe 1 e 273 na equipe 2. A seleção desses usuários ocorreu aleatoriamente, por meio de sorteio dos domicílios. Entretanto, houve perdas na amostra porque algumas pessoas optaram por não participar da pesquisa e outras não estavam em suas residências no momento da coleta de dados, de modo que fizeram parte do estudo 386 usuários, sendo 224 da equipe 1 e 162 da equipe 2 .

Foi utilizado como instrumento de coleta de dados um questionário com questões fechadas, mediante entrevista realizada pelos pesquisadores aos respondentes. As variáveis foram: perfil dos entrevistados; a prática de atividade física; se a pratica, que tipo é realizado; a frequência; a duração e o local; e, se não realiza, o porquê de não fazê-la. A coleta de dados ocorreu entre os meses de outubro de 2010 e fevereiro de 2011, nos domicílios dos usuários, mediante sua disponibilidade.

Os dados foram agrupados com o auxílio do programa Microsoft Excel 2007, com distribuição absoluta e relativa dos dados obtidos pelos questionários.

O desenvolvimento do estudo seguiu as diretrizes emanadas da Resolução no 196/96 do Conselho Nacional de Saúde, que regulamenta as normas aplicadas a pesquisas que envolvem, direta ou indiretamente, seres humanos. 
Precedendo o trabalho de campo, o projeto de pesquisa foi submetido à avaliação do Comitê de Ética em Pesquisa da Universidade Estadual da Paraíba com emissão do parecer favorável, apresentando o Certificado de Apresentação para Apreciação Ética (CAAE) de número 4114.0.000.133-10. A coleta ocorreu nas residências dos usuários cadastrados após serem informados da finalidade da pesquisa e terem assinado o Termo de Compromisso Livre e Esclarecido.

\section{Resultados e Discussão}

Participaram da pesquisa 386 usuários, sendo $319(82,6 \%)$ do sexo feminino e apenas $67(17,1 \%)$ do sexo masculino. Os dados apresentados revelam um grande número de mulheres participantes da pesquisa, entretanto, isto pode ser explicado pelo horário da coleta de dados da pesquisa - manhã e tarde. $\mathrm{Na}$ maioria das vezes, os homens estavam trabalhando e as mulheres permaneciam em casa para realizar atividades domésticas.

Quanto à escolaridade, observou-se que 155 $(40,2 \%)$ apresentavam o ensino fundamental incompleto, $90(23,3 \%)$ ensino médio completo, 57 $(14,8 \%)$ ensino fundamental completo, $42(10,9 \%)$ ensino médio incompleto, 27 (7\%) ensino superior e 15 (3,8\%) não eram alfabetizados.

Quando os usuários da UBSF Malvinas I foram questionados quanto à prática de exercício físico, 275 (71,24\%) disseram que não praticam e 111 (28,76\%) responderam que sim. Este dado é preocupante, visto que o cuidado, essencial à sobrevivência humana, está sendo cada vez mais atribuído aos profissionais e serviços de saúde, eximindo o sujeito de seu autocuidado $^{17}$. A baixa adesão dos usuários à atividade física também foi referido em outro estudo que revelou percentual de adesão de $26 \%{ }^{18}$.

Ao cruzar os dados de nível de escolaridade e a prática de atividade física entre todos os 386 participantes e observar a adesão em cada estrato, verificouse que entre os não alfabetizados não houve adesão à atividade física. Entre os 155 que cursaram o ensino fundamental incompleto, 35 (22,5\%) aderiram; dos 57 que concluíram o ensino fundamental, apenas 12 (21\%). Dos 42 com ensino médio incompleto, 32 (76\%) informaram adesão à atividade física; entre os 90 que cursaram o ensino médio completo, apenas $11(12 \%)$ realizavam atividade física e 21 (78\%) dos 27 que cursaram o ensino superior afirmaram adesão.

$\mathrm{Na}$ amostra do estudo, o estrato de escolaridade que apresentou maior adesão foi o de ensino superior, seguido do ensino médio incompleto. A relação de níveis socioeconômicos e escolaridade são evidenciadas em diversos estudos, destacando que as pessoas com melhores condições financeiras e grau de estudo têm uma maior facilidade ao acesso dos serviços de saúde e, consequentemente, as informações e cuidados de saúde ${ }^{7}$. Outra justificativa seria que as pessoas com uma formação educacional maior possuem um maior tempo livre para realizarem atividade física, visto que, os indivíduos sem essa formação estão envolvidos com atividades domésticas, principalmente as mulheres ${ }^{19}$.

A melhora dessas estatísticas em prol da atividade física pode ser alcançada pelo aconselhamento do profissional de saúde, por campanhas de incentivo e pelo desenvolvimento de grupos de atividade física formados pelos usuários das unidades de saúde. As campanhas atuariam como meio de informação mais influente, porque poderiam responder às dúvidas das pessoas, como também poderiam informar os benefícios do exercício para a saúde de uma forma mais clara, ou seja, mais acessível. Já os grupos serviriam como meio para troca de experiências entres os participantes ${ }^{20}$.

A diminuição na prática de exercícios físicos é verificada em todo o mundo, apesar da sua importância para a promoção e prevenção da saúde tanto física quanto mental. No Brasil, embora a quantidade de estudos para pesquisar o nível de atividade física na população seja pequena, os trabalhos desenvolvidos até então mostram uma tendência para inatividade física e de lazer ${ }^{19,21}$.

Quanto aos motivos de não praticar atividade física, $105(38,2 \%)$ responderam que era a falta de tempo que os impedia, 22 (8\%) não praticava devido a complicações de doenças, $21(7,63 \%)$ por dores e cansaço e 127 (46,17\%) disseram outros motivos, alegando, inclusive, a própria indolência.

Os vários motivos que os participantes apresentaram como justificativa para a não realização da atividade física revela que a qualidade de vida proporcionada pelo exercício, amplamente divulgada pela mídia, está sendo deixada de lado, especialmente pela falta de tempo, como revela a pesquisa. Além disso, esse resultado indica que a melhora da qualidade de vida por meio da prática de atividade física ainda não adquiriu a importância necessária entre os participantes.

Entre as pessoas que realizavam atividades físicas, a caminhada se sobressaiu com 101 (91\%) indivíduos e apenas $10(9 \%)$ mencionaram outros tipos de atividades. Essa porcentagem mostra que, mesmo sem ambiente apropriado, como as ruas locais com pavimentação ruim, com intenso movimento automobilístico e sem material específico, alguns participantes estão caminhando para melhorar a sua qualidade de vida ${ }^{22}$.

Quanto à frequência dos exercícios físicos, 62 $(55,88 \%)$ afirmaram realizar mais de três vezes por semana; 31 (27,92\%), três vezes por semana; 10 (9\%), duas vezes por semana e $8(7,2 \%)$, apenas uma vez por semana. Entre os praticantes, a maioria é consciente que estas devem ser exercidas com maior frequência, com recomendação de no mínimo três dias intercalados por semana, sendo o ideal todos os dias. 
Nesse sentido, a equipe deve buscar, por meio do diálogo e da educação em saúde, pactuar com os usuários modos de cuidar da saúde, compartilhando a responsabilidade com o cuidado ${ }^{23-25}$.

Em relação ao tempo gasto para a realização do exercício físico, 80 (72,03\%) entrevistados responderam entre 30 e 60 minutos; 11 (9,9\%), mais de 60 minutos; 11 (9,90\%), entre 20 e 30 minutos e 9 $(8,1 \%)$, menos de 20 minutos.

O tempo disponibilizado pelos entrevistados para a prática da atividade física, de 30 a 60 minutos diários, revela que aqueles que já a praticam conseguem manter-se em constante atividade, talvez por perceber os seus vários benefícios, e seguem as recomendações para a promoção e manutenção da saúde em adultos e idosos, que preconizam a realização de, no mínimo, 30 minutos de atividades físicas aeróbicas de intensidade baixa a moderada de 3 a 5 vezes por semana ${ }^{26}$.

\section{ConClusão}

O estudo alcançou seu objetivo à medida que definiu o perfil dos usuários da unidade de saúde analisada, particularizando tal realidade. Isso indica que, para cada unidade de saúde, os profissionais, ao incentivarem a prática da atividade física, devem fazê-lo pautados na necessidade e características da população atendida, para que a implantação de um programa desta natureza seja uma iniciativa viável tanto do ponto de vista social quanto de promoção da saúde.

Em relação àqueles que não praticam exercícios, a maioria respondeu que não o faz por falta de tempo, o que é uma preocupação relevante, pois, muitas vezes, a importância dessa prática não é levada em consideração. Por isso, os indivíduos não reservam um período de tempo apropriado para sua realização, sendo necessário conscientizar a população dos benefícios da atividade física, como a redução de agravos à saúde e uma melhor qualidade de vida.

A prática regular de atividade física emerge como um importante recurso a ser incorporado pela Atenção Básica em suas ações de promoção da saúde, uma vez que traz uma variedade de benefícios para a população, como prevenção de doenças e agravos não transmissíveis. Portanto, considera-se a necessidade de se incluir a atividade física, além das medidas gerais de saúde, para promover a saúde da população, reduzir o risco de desenvolvimento de doenças e melhorar a qualidade de vida.

O estudo apresentou algumas limitações, sendo a principal delas a aplicação da entrevista a um número reduzido de usuários do sexo masculino devido ao fato de não se encontrarem em seus domicílios nos turnos da coleta de dados, tendendo a considerar os resultados encontrados apenas para aquelas do sexo feminino. Este fato também impossibilitou o cruzamento de dados relacionando a atividade física à questão de gênero.

\section{REFERÊNCIAS}

1.Pitanga FJG. Epidemiologia, atividade física e saúde. Rev Bras Ciên e Mov. [online] 2002; 10 (3).

2.Pucci GCMF, Rech CR, Fermino RC, Reis RS. Associação entre atividade física e qualidade de vida em adultos. Rev Saúde Pública. [online] 2012; 46(1): 166-79.

3.Codogno JS, Fernandes RA, Monteiro HL. Prática de atividades físicas e custo do tratamento ambulatorial de diabéticos tipo 2 atendidos em unidade básica de saúde. Arq Bras Endocrinol Metab. [online] 2012; 56(1): 6-11. 4.Fernandes NP, Bezerra CRM, Neto JS, Batista VLM, Pedrosa CCLM. A Prática do Exercício Físico para melhoria da qualidade de vida e controle da Hipertensão Arterial na terceira idade. Rev Ciênc Saúde Nova Esperança. [online] 2013; 11(3): 60-6.

5.Ramalho JRO, Lopes ACS, Toledo MTT, Peixoto SV. Nível de atividade física e fatores associados ao sedentarismo em usuários de uma Unidade Básica de Saúde em Belo Horizonte, Minas Gerais. Rev Min Enferm. [online] 2014; 18(2): 426-32.

6.Zamai CA, Bankoff ADP, Silva JF, David LT, Silva TTR, Dias $\mathrm{C}$, et al. Concepções sobre a prática de atividades físicas e saúde na educação física e esportes. Rev Facul Educ Fis da UNICAMP. [online] 2009; 7 (3).

7.Palma A. Atividade física processo e saúde-doença e condições sócio-econômicas: uma revisão da literatura. Rev paul Educ Fis. [online] 2000; 14 (1).

8. Wing RR, Jakicic JM. Mudando o estilo de vida: de sedentarismo a ativo. Atividade física e obesidade. 2003; 19: 439.

9. Weiss CV, Oliveira MM, Weiss CV, Franzman UT, Coimbra VCC, Alves PF. A importância da caminhada na perspectiva da equipe de redutores de. J Nurs Health, Pelotas (RS). [online] 2012 2(1): 44-9.

10.Matsudo SM, Matsudo VKR, Barros Neto TL. Atividade física e envelhecimento: aspectos epidemiológicos. Rev Bras Med Esporte. [online] 2001; 7 (1).

11.Amodeo C, Lima NKC. Tratamento não medicamentoso da hipertensão arterial. Medicina. 1996; 29: 239-43. 12.Mello MT, Fernandez AC, Tufik S. Levantamento epidemiológico da prática de atividade física na cidade de São Paulo. Rev Bras Med Esporte. [online] 2000; 6:119-24.

13.Guyton AC, Hall JE. Fisiologia médica. 11 a ed. Rio de Janeiro: Guanabara Koogan; 2006.

14.Freitas R, Freire WJ. Prática de atividade física por adolescentes de Fortaleza, CE, Brasil. Rev Bras Enferm. [online] 2010; 63: 410-5.

15.Benedetti TRB, Borges LJ, Petroski EL, Gonçalves LHT. Atividade física e estado de saúde mental de idosos. Rev Saude Publica. [online]. 2008; 42 (2).

16.Ciolac EG. Efeito do exercício físico contínuo versus intervalado sobre pressão arterial, rigidez arterial e qualidade de vida em pacientes hipertensos [dissertação]. São Paulo: Universidade de São Paulo, Faculdade de Medicina; 2006 [citado em 24 set 2012]. Disponível em: http://www.teses.usp.br/teses/disponiveis/5/5160/ tde-07082006-104223/. 
17.Felipe GF, Silveira LC, Moreira TMM, Freitas MC. Presença implicada e em reserva do enfermeiro na educação em saúde à pessoa com hipertensão. Rev enferm UERJ. 2012; 20: 45-9.

18.Santos RP, Horta PM, Souza CS, Santos CA, Oliveira HBS, Almeida LMR, Santos LC. Aconselhamento sobre alimentação e atividade física: prática e adesão de usuários da atenção primária. Rev Gaúcha Enferm. [online] 2012; 33(4): 14-21.

19.Salles CR. Gênero e prática de atividade física de lazer. Cad Saúde Pública. 2003; 19: 325-33.

20.Warschauer M, D’Urso L. Ambiência e formação de grupo em programas de caminhada. Saúde soc. [online] 200; 18 (2): 104-7.

21.Knuth AG, Malta DC, Dumith SC, Pereira CA, Morais NOL, Temporão JG, et al. Prática de atividade física e sedentarismo em brasileiros: resultados da Pesquisa Nacional por Amostra de Domicílios (PNAD) 2008. Ciênc Saúde Coletiva. [online] 2011; 16(9): 3697-705
22.Monteiro WD, Araújo CGS. Transição caminhadacorrida: considerações fisiológicas e perspectivas para estudos futuros. Rev Bras Med Esporte. [online] 2001; 7 (6): 207-22.

23.Nery AA, Carvalho GR, Santos FPA, Nascimento MS, Rodrigues, VP. Saúde da Família: visão dos usuários. Rev enferm UERJ. 2011; 19: 397-402.

24.Jones DE, Carson KA, Bleich SN, Cooper LA. Patient trust in physicians and adoption of lifestyle behaviors to control high blood pressure. Patient Educ Couns 2012. [online] 89 (1): 57-62.

25.Moreira RP, Guedes NG, Lopes MVO, Cavalcante TF, Araújo TL. Diagnóstico de enfermagem estilo de vida sedentário: validação por especialistas. Texto contexto - enferm. [online]. 2014, 23 (3): 547-54.

26.Kokubun E. Programa de atividade física em unidades básicas de saúde: relato de experiência no município de Rio Claro. Revista Brasileira de Atividade Física \& Saúde. [online] 2007; 12: 45-50. 